\title{
Atypical case of subconjunctival hemorrhage
}

\author{
Joaquín I Oporto*, Rafael Velasco, Antonia Mori and Camila Olivares \\ Pontificia Universidad Católica de Chile, Santiago, Chile
}

\begin{abstract}
Background: Red eye syndrome is the most common cause for primary care ophthalmological consults. One of the causes is subconjunctival hemorrhage, which is often asymptomatic. We now report a case of subconjunctival hemorrhage associated to atypical symptoms.

Case presentation: A 50-year-old woman presents to the primary care setting with a two-day history of right eye redness. Direct clinical questioning reveals the presence of photophobia and diminished visual acuity. Both symptoms were later confirmed on clinical examination. The appearance of the eye was confirmatory for subconjunctival hemorrhage.
\end{abstract}

Conclusions: Subconjunctival hemorrhage may present with symptoms of other eye-threatening conditions. Clinical examination is critical to distinguish between those causes and a benign subconjunctival hemorrhage.

\section{Introduction}

Ophthalmological consults account for approximately $2 \%$ to $3 \%$ of primary-care consults [1], being the red-eye syndrome the most frequent amongst them [2]. Subconjunctival hemorrhage usually occurs in patients with poorly-controlled arterial hypertension and patients under the effects of anticoagulant drugs [3], especially after performing the Valsalva maneuver [4]. This pathology is generally asymptomatic, unilateral and the diagnosis is usually made solely based on clinical examination [5].

\section{Case report}

A 50-year-old woman, with a history of arterial hypertension in regular control with 50 milligrams of losartan a day, and an unremarkable past ophthalmological history, attended to a primarycare consult referring a two-day episode of redness in the right eye. It began after lifting heavy boxes during manual housework. After direct clinical questioning, she referred photophobia, diminished visual acuity and foreign body sensation in the right eye, all of which had started the previous day. She denied secretion, pain or other symptoms. Physical examination revealed a localized hemorrhage beneath the conjunctiva in the right eye (Figure 1) with no corneal lesions. Her best corrected visual acuity was 20/30 in the right eye and 20/20 in the left eye. Eyelid eversion revealed no foreign bodies. Both pupils were symmetrical and photo motor reflexes, accommodation reflexes and oculomotility remained unaltered. Direct photophobia was confirmed, but consensual photophobia was ruled out.

Under the impression of a subconjunctival hemorrhage, considering the typical clinical appearance, the patient was educated about the pathology and possible red-flag symptoms. She was sent home and cited for the next day, when she persisted with nonobjectifiable diminished visual acuity. A week later, hemorrhaging became less apparent and she completely asymptomatic. By the second week, the full extent of the hemorrhage and the symptoms were gone.

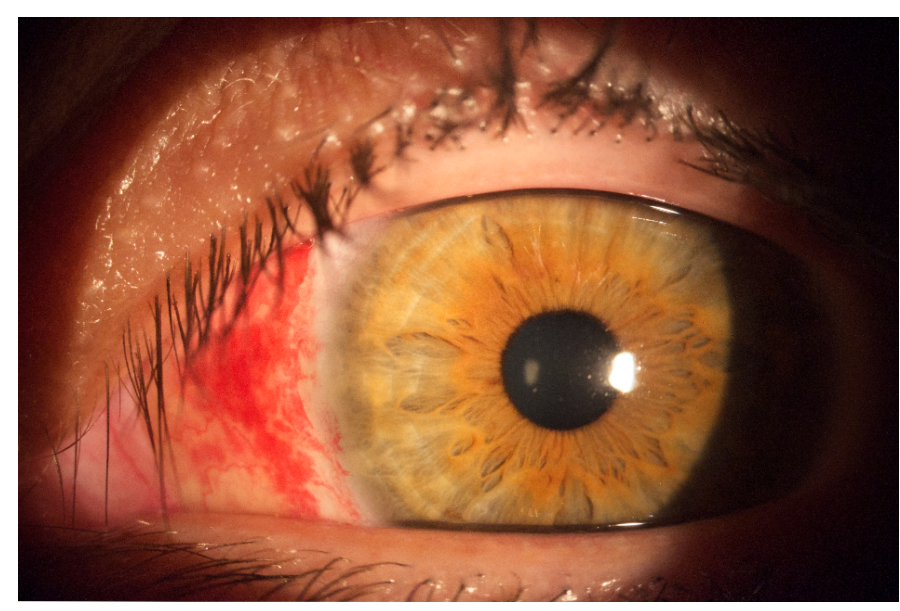

Figure 1. Patient's right eye showing a subconjuntival hemorrhage

\section{Discussion}

Red-eye syndrome is usually divided into benign causes, which can be managed by the general practitioner and primary-care physician, from other more serious and eye-threatening conditions, which must be promptly referred to a specialist. To discern between these two, clinical findings are of foremost importance, given that the presence of red-flag signs or symptoms usually indicate a deeper or serious redeye. The three cardinal symptoms usually taught in medical schools to differentiate between different causes of red-eye are pain, diminished visual acuity and photophobia [6]. Nonetheless, likelihood ratio (LR)

${ }^{\star}$ Correspondence to: Joaquín I. Oporto, Pontificia Universidad Católica de Chile, Santiago, Chile, Tel: +56998429745; E-mail: jnoporto@uc.cl

Key words: primary care; red eye; subconjunctival hemorrhage

Received: December 11, 2019; Accepted: December 18, 2019; Published: December 20, 2019 
scores for these three symptoms have yet to be further studied. Another group of authors proposed that the presence of markedly red-eye, a foreign object or history of trauma should also be discarded [7].

In our patient's case, even though she presented with red-flag symptoms (diminished visual acuity and direct photophobia), the rest of the exam was normal. Clinical examination and aspect of the orbit turned out to be characteristic of subconjunctival hemorrhage. Taking into account the patient presenting with direct photophobia (LR 8.3), but no indirect photophobia or near synkinesis test (LR 28.8 and 21.4 , respectively) [6], we decided to treat de patient and abstain from referring her to a specialist.

To our knowledge, there are no reported cases of subconjunctival hemorrhage associated to red-flag symptoms. This turns out to be relevant given the uncertainty future general practitioners, primary care physicians and emergency department doctors may face with similar clinical manifestations. This may avoid unnecessary referrals to the ophthalmologists and the tertiary healthcare system in the future. In this case, the most important aspect was the typical history reported by the patient and characteristic clinical findings observed at the physical examination.

Another relevant aspect to highlight is the need for continuity in patient-care. In this case, only non-measurable lessening of the visual acuity was persistent, with all other symptoms being gone by the next day. During the following weeks, the clinical episode subsided and followed its natural course, with hemorrhage disappearing by the second week. Patient follow-up helped to avoid possible complications or the addition of new symptoms to the episode, changing the original diagnosis and the course of treatment.

\section{Conclusions}

Although subconjunctival hemorrhage is described as an asymptomatic, acute onset red eye, this case confirms that it might not always be the same. Symptoms can be confusing, so clinical appreciation is of upmost importance. Patient follow-up is also critical in order to avoid committing eye-threatening mistakes.

\section{Authorship}

All listed authors have made substantive intellectual contributions to the case follow-up, manuscript design and bibliographic research.

\section{Acknowledgments}

None.

\section{Financial support}

None.

\section{Ethical aspects}

The patient offered a verbal informed consent to this publication and anonymity was assured.

\section{Conflicts of interests}

The authors declare no conflicts of interests.

\section{References}

1. Pflipsen M, Massaquoi M, Wolf S (2016) Evaluation of the Painful Eye. Am Fam Physician 93: 991-998.

2. Cronau H, Kankanala RR, Mauger T (2010) Diagnosis and management of red eye in primary care. Am Fam Physician 81: 137-144.

3. Tarlan B, Kiratli H (2013) Subconjunctival hemorrhage: risk factors and potentia indicators. Clin Ophthalmol 7: 1163-70.

4. Oporto J, Zuazo F, Valdes G, Contreras X (2011) Red eye syndrome in primary care diagnosis and management. Chilean Journal of Family Medicine 10: 7-11.

5. Leibowitz HM (2000) The red eye. N Engl J Med 343: 345-351.

6. Narayana S, McGee S (2015) Bedside Diagnosis of the 'Red Eye': A systematic review. Am J Med 128: 1220-1224.e1.

7. Kilduff C, Lois C (2016) Red eyes and red flags: Improving ophthalmic assessment and referral in primary care. BMJ 5: u211608.w4680.

Copyright: (C2019 Oporto JI. This is an open-access article distributed under the terms of the Creative Commons Attribution License, which permits unrestricted use, distribution, and reproduction in any medium, provided the original author and source are credited. 